\title{
Letter
}

\section{The brain seduction: the public perception of neuroscience}

\author{
Donato Ramani
}

\begin{abstract}
The increasing number of magazine covers dedicated to brain studies and the success of magazines and scientific journals entirely dedicated to brain and mind indicate a strong interest on these themes. This interest is clearly surpassing the boundaries of scientific and medical researches and applications and underlines an engagement of the general public, too. This phenomenon appears to be enhanced by the increasing number of basic researches focusing on non-health-related fMRI studies, investigating aspects of personality as emotions, will, personal values and beliefs, selfidentity and behaviour. The broad coverage by the media raises some central questions related to the complexity of researches, the intrinsic limits of these technologies, the results' interpretative boundaries, factors which are crucial to properly understand the studies' value. In case of an incomplete communication, if those fundamental interpretative elements are not well understood, we could register a misinterpretation in the public perception of the studies that opens new compelling questions. As already observed in the past debates on science and technologies applications, in this case, too, we assist to a communicative problem that set against scientific community on one side and media, on the other. Focusing our attention, in particular, on the debate on fMRI, taken as a good model, in the present letter we will investigate the most interesting aspects of the current discussion on neuroscience and neuroscience public perception. This analysis was performed as one of the bid - brains in dialogue - activities (www.neuromedia.eu). bid is a three year project supported by the European Commission under the 7th Framework Program and coordinated by Sissa, the International School for Advanced Studies of Trieste, aimed at fostering dialogue between science and society on the new challenges coming from neuroscience.
\end{abstract}

\section{Neuroscience in the media}

"The problem right now with imaging is that doing experiments right is really, really hard, but getting pictures out is really easy" declared Steven Petersen from Washington University in St. Louis, Missouri, in Greg Miller's article titled Growing Pains for fMRI. ${ }^{1}$ In recent years fMRI Functional Magnetic Resonance Imaging and PET (Positron Emission Tomography) have evolved, becoming key tools in neuroscientific studies, with important clinical and research applications. Today, in the clinical domain, those technologies are commonly used for diagnosis of tumours or lesions in the brain nervous system, carrying hope for future applications in drug development monitoring and early diagnosis in a wide range of neurological diseases (first of all in the neurodegenerative ones, such as Parkinson and Alzheimer). While hopes and good results are emerging, ethical and social implications of neuroimaging application are becoming more and more pressing. The sound connection of those issues to neuroscience research press coverage, neuroscience dissemination and some aspects of basic research, too, look evident.

The increasing number of magazine covers dedicated to brain studies and the success of magazines and scientific journals entirely dedicated to brain and mind indicate a strong interest on these themes. This interest is clearly surpassing the boundaries of scientific and medical researches and applications and underlines an engagement of the general public, too. This phenomenon appears to be enhanced by the increasing number of basic researches focusing on non-health-related fMRI studies, investigating aspects of personality as emotions, will, personal values and beliefs, self-identity and behaviour.

The broad coverage by the media raises some central questions summarized in Peterson's opinion quoted at the beginning of this paragraph. Peterson's quote relates directly to the complexity of researches, the intrinsic limits of these technologies, the results' interpretative boundaries, factors which 
are crucial to properly understand the studies' value. In case of an incomplete communication, if those fundamental interpretative elements are not well understood, we could register a misinterpretation in the public perception of the studies that opens new compelling questions. In fMRI in the public eye, ${ }^{2}$ published on Nature Reviews in 2005, the three authors, Eric Racine, Ofek Bar-Ilan and Judy Illes, underline this urgency. In their study they investigate how "both neuroscience and the media shape the social understanding of fundamental aspects of our reality" and frame their argument in three trends. Those trends come from their fMRI press coverage observation. The three concepts identified, called neuro-realism, neuro-essentialism and neuro-policy, well resume the major points of discussions around ethical, social and legal concerns related to neuroscience.

The three concepts and the interaction among them, as the authors write, "encompass lay perception of reality, subjectivity and policy making" and "combined with both hope and leap of faith about the meaning of the data across the lifespan; contribute to public appreciation of the benefits and risks of functional neuroimaging". Those interactions deeply influence the science evolution, too, in a strong connection between science and society.

Neurorealism, the first concept explored, is described as the phenomenon for which the evidences coming from neuroimaging become "real, objective or effective" in the public eye. A sort of "visual proof", "despite the enormous complexity of data acquisition and image processing", and "a final proof", too, of what people always felt "real" and "true" but, up to now, nobody has been able to visualize: pain, feelings, fear, pleasure, even faith. In other words, fMRI could work as validation of what has always been considered evanescent and impalpable. In such a way "neuro-realism reflects the uncritical way in which an fMRI investigation can be taken as validation or invalidation of our ordinary view of world."

The second concept is summarized in the "neuro-essentialism" definition that reflects how "fMRI research can be depicted as equating subjectivity and personal identity to the brain." In this vision brain would become the synonym of identity and personality, a word used "as a shortcut for more global concepts". Looking at the brain, we should be able to catch the true essence of personality (i.e. "brain cannot lie"), the origin of individual differences (differences between man and woman, homosexuality, vices etc.) and so on.

The third concept, "neuropolicy", describes the political use of neuroimaging findings, used to promote specific issues included in the agendas of religious groups, parties, etc. The neuroimaging "visual proof" can have a strong impact on people, supporting one thesis instead of the opposite one. In this sense, a clarifying example comes from the last U.S. presidential election. In November 2007 the New York Times published a column describing a study in which 20 undecided voters had their brain activity scanned by fMRI while the images and videos of candidates were showed them. The article publication had a great echo animating a big debate: "It was really closer to astrology than it was to real science" was the comment of Russell Poldrack of UCLA (University of California at Los Angeles).

The New York Times case can well clarify the different aspects of neuroimaging applications. As Poldrack quoted: "It epitomized everything that a lot of us feel is wrong about where certain parts of the field are going, which is: throw someone in a scanner and tell a story about it".

Across the media and the scientific worlds, a really interesting phenomenon is emerging. On one side we have media that fish in the scientific publications, looking for interesting news (i.e. news that could catch the interest of readers) related to neuroscience. As a consequence, researches that investigate people emotional state will, truth, or specific human attributes (love, faith, morality) find a great media coverage regardless of the findings scientific relevance. On the other side researchers and neuroscientists underline the limitations of brain imaging techniques and the possible risks of data misinterpretation. In this context, an old word, "phrenology", is surprisingly coming back in the neuroscientists' quotes. "Neophrenology" could be, in their opinion, a possible bad consequence of bad communication: "People will start to see fMRI as neophrenology, just telling stories and not giving explanations" said Poldrack. In the debate, the focal point is what the activation of specific areas really means for the scientist and for the public. What scientists see in specific brain activations, and the conclusions they can draw from those, could be so much different that some reflections on the impact of media coverage on general perception are needed.

As we have already written, in the neuroscience public perception investigation, fMRI surely represents a good model for discussion. In this context, "media coverage constitutes one important pathway to assess what the issues surrounding fMRI are and how the technology is portrayed outside the peerreviewed literature."3 
An other important study by Eric Racine, Ofek Bar-Ilan and Judy Illes, titled Brain Imaging, a decade of coverage in the print media, re-analyzed articles published from 1991 to 2004 using the LexisNexisAcademic database with the keywords "Neuro AND Imaging" and "functional magnetic resonance imaging OR fMRI". The research was focused on general news (major newspapers, magazines and journals), medical news (medical and health news), university news and legal news. The researchers analyzed 132 articles: 60\% represented general source articles, 53 (40\%) represented specialized sources.

Health related researches featured neurodegenerative diseases such as Alzheimer's and Parkinson's diseases and psychiatric conditions such as depression and schizophrenia. Coverage of non health-related researches focused principally on higher order cognitive systems such as decision making and motivation, other cognitive behaviour that have emotional and potential social impacts and basic cognitive systems such as attention and memory. In half of the articles perspectives of health-related benefits of fMRI were featured. In many fewer non-health prospects were found. $79 \%$ of articles were uncritical in tone, ranging from optimistic to neutral. Just $21 \%$ were balanced or critical. The results of quantitative analysis related to Tone and Concerns identified in Press Coverage are reported in the table 1:

\begin{tabular}{|l|l|l|l|}
\hline Code & $\begin{array}{l}\text { Health-related } \\
\text { Research }\end{array}$ & $\begin{array}{l}\text { Non-Health-Related } \\
\text { Research }\end{array}$ & Both \\
\hline Tone & & & \\
\hline Uncritical & 91 & 78 & 63 \\
\hline Balanced & 9 & 14 & 30 \\
\hline Critical & 0 & 9 & 7 \\
\hline Issues & & & 41 \\
\hline At least one issue & 9 & 24 & 30 \\
\hline Scientific issue & 9 & 21 & 19 \\
\hline Ethical issue & 0 & 5 & \\
\hline Benefits & & & 70 \\
\hline Health benefit & 63 & 29 & 19 \\
\hline Non-health-related benefit & 2 & 19 & \\
\hline
\end{tabular}

Table 1. Tone and Concerns identified in Press Coverage. ${ }^{3}$

In a qualitative research, aimed to enrich the understanding, limitations of technology were found in nineteen of the articles. Three classes of limitations were found: 1) "emphasis on the preliminary nature of findings or their limited generalizability by neuroimagers themselves" 2) "Challenges to the basic assumption that levels of blood oxygenation are indicators of neuronal activity" 3) "Worries associated with the wider social usefulness of fMRI research and findings".

Validity of fMRI techniques was dealt with in a minority of articles that focused on the risks of and concerns about poor scientific research design or lack of standardized practices. Interpretation issues intended as over interpretation of results related to the possibility of correlating brain activation to neurocognitive function or as the wider meaning of MRI findings in the public arena - were found in ten articles. Ethical concerns were found in 6 articles facing the "Human nature" matter (in relation, in particular, to neuromarketing) and in few other pieces where themes like confidentiality, privacy issues, protection of human subjects enrolled in research were discussed.

In the article discussion, authors pointed out the general media optimism for this technology. In particular "clinical research is presented more optimistically than nonclinical research, and scientific concerns are featured more often than the ethical ones." At the same time, it is interesting to note that press coverage, in general, is great for non-health-related phenomena, in particular higher order cognition, emotion, and social behaviour although health benefits are more emphasized.

Looking at those data, disequilibrium emerges. On one side the interest for non-health brain imaging techniques applications is greater than for the health ones. On the other side, health related phenomena seem to have a better consideration. Some researchers ${ }^{4}$ suggested that health content may be used to facilitate social acceptance of fMRI. In effect, there are no doubts that those health benefits are among the most appreciated outcomes of scientific research. 
In any case, authors noted, although "in contrast to the optimism for fMRI research, we did find substantial content related to specific scientific and ethical concerns", "the presence of ethical issue in fMRI reporting is still lower, however, than media coverage of genomics and genetics. [...]. This comparison sustains the point of view that the issues of neuroscience have not yet been brought to the public eye as frequently as issues of genomics."

\section{Studies on neuroscience perception}

To understand the possible impact of media coverage on general perception of neuroscience some interesting studies have been performed. Psychologists David Mc Cabe of Colorado State University and Alan Castel of the University of California in their study published on Cognition journal, for example, examined "whether brain images actually do have a particularly powerful persuasive influence on the perceived credibility of cognitive neuroscience data." Using brain images to represent brain activity confers a big deal of scientific credibility to studies of cognition: is this thesis, suggested by many researchers and media, ${ }^{6}$ true?

The two researchers asked to 156 undergraduate students to evaluate some mock news articles describing brain imaging studies. All the researches submitted to the students' evaluation were fake and the conclusions they reported clearly fictitious. In the authors words: "The articles made claims that were not necessitated by the data (e.g., reverse inference errors), giving participants some basis for scepticism in their ratings." Just an example: in the article entitled, 'Watching TV is Related to Math Ability', it was concluded that because watching television and completing arithmetic problems both led to activation in the temporal lobe, watching television improved math skills. The other two articles, entitled 'Meditation Enhances Creative Thought', and, 'Playing Video Games Benefits Attention', also included errors in scientific reasoning, and preceded the 'Watching TV' one. The fictional articles summarizing cognitive neuroscience research either included no image, a brain image, or a bar graph depicting the critical results.

After reading the article, participants were asked to rate the soundness of the scientific reasoning in the article. In the first experiment, three statements were submitted to students: (1) The article was well written, (2) The title was a good description of the results, and (3) The scientific reasoning in the article made sense. Responses were made on a four-point Likert scale, with response options including "'strongly disagree", “disagree", "'agree', and "strongly agree"' (coded 1, 2, 3, or 4, respectively, for purposes of data analysis). The students' answers evidence that a positive relationship exists between brain representation and ratings. As authors write in the paper: including brain images, a seemingly direct physical representation of brain activity, with summaries of fictional cognitive neuroscience data, increased ratings of scientific reasoning for those summaries. To test the hypothesis that positive ratings for brain imaging be connected to the visual complexity a second experiment was performed. In this second test "participants were presented with articles that were accompanied by brain images and others that were accompanied by topographical maps of brain activation". In their representation of brain activity, the topographical maps were similar to the brain images although an important and distinguishing factor was previewed: in fact, "These maps are not typically used in the popular press, and presumably are not as easily identified as representing a brain". In this experiment just the statement the scientific reasoning in the article made sense was rated. The test result looks really interesting since "texts accompanied by a brain image were given higher ratings of scientific reasoning than those accompanied by a topographical map". Since both images were visually complex, the higher score obtained by brain imaging images evidences that some other elements should be considered.

On the basis of these results we can conclude "that's an image matter" and, at the same time, that each type of image has a specific communicative potential. In the third experiment, researchers used real news entitled Brain Scans Can Detect Criminals. Two statements were rated by students: (1) Do you agree or disagree that the title, Brain Scans Can Detect Criminals, is a good summary of the results? And (2) Do you agree or disagree with the conclusion that brain imaging can be used as a lie detector? Moreover, "for half the participants the last paragraph of the article included a quotation of a researcher criticizing the conclusion that brain imaging could be used as a lie detector to detect criminal activity in the real world, but for the other half this text was omitted". Once again the results of this third test testimony the image power, able to convince the lie detection potential of brain imaging.

As we can see, in the case of fictional articles as well as in the real ones the tests' results didn't substantially differ: brain images have a particular persuasive potential in conferring credibility to 
neuroscience data. In general public perception they seem to represent a strong and intuitive shortcut for brain functions understanding. As the authors write in their article "This sort of visual evidence of physical systems at work, which is typical of "harder" sciences like physics and chemistry, is not typically apparent in studies of cognition, where the evidence for cognitive processes is indirect, by nature." The fMRI's technique based on changes in brain oxygenation, and the indirect results this technique gives to researchers, are not considered by the readers in their ratings. In those tests neither the quality of the research nor the researchers' affiliations seem to influence the public opinion. On the contrary, the experiments results "lend support to the notion that part of the scientific credibility of brain imaging as a research technique lies in the images themselves."

In his article on nova, ${ }^{7}$ scientific supplement of Il Sole 24 Ore Italian newspaper, professor Carlo Umiltà, from Padua University, author with Paolo Legrenzi of a book dedicated to neuroscience entitled Neuro-Mania, writes: "The human brain, as that one of other primates, is largely dedicated to visual information elaboration. That is why visual evidence is so persuasive." Underlying the numerous steps requested to obtain a specific visual result, at the end of his article Umiltà asks: "Would coloured images be so convincing even if readers knew those images are the result of an elaborate sequence of "cleaning actions", each one characterized by a not insignificant error probability?" Paolo Legrenzi, Neuro-mania's co-author, in another piece writes that thanks to neuroscience an old dream is coming true. Legrenzi directly refers to the "Man a machine" concept, coming from eighteenth century La Metrie's manifesto. In his opinion, the neuroscience debate is just the last example of a never cancelled reductionist temptation that is the thrust to reduce the essence of human being to simple mechanisms, and to look at the human behaviours, thoughts, emotions as a direct result of those. Or, in other words: simple origins for complicated and multiform expressions. People seem "Born to believe", is the Legrenzi statement.

With respect to public perception, Explanation seems to be the key word. That is what Weisberg et al. ${ }^{8}$ claim in their article entitled The Seductive Allure of Neuroscience Explanations. "People often believe explanations because they find them intuitively satisfying not because they are accurate. 9 " It is interesting to note that analysing the different aspects of neuroscience images' perception, we are crossing the boundaries of neuroscience entering in psychology. Neuroscience information, in this sense, works as a marker of good explanation, regardless of the information content. "People may therefore uncritically accept any explanation containing neuroscience information, even in cases when the neuroscience information is irrelevant to the logic of the explanation".

As the authors illustrate, many researches have shown that people have difficulty reasoning about explanations. For example:

- People tend to consider and rate longer explanation as more similar to the experts' one e $^{10}$

- People fail to recognize circularity ${ }^{11}$

- People are quite unaware of the limits of their own abilities to explain a variety of phenomena ${ }^{12}$

- People can be influenced by teleological explanations when these are not warranted, as in cases where a nonteleological process, such as natural selection or erosion, is actually implicated. ${ }^{13,14}$

The tests used by researchers aimed to investigate people's judgement of explanations that do or do not contain neuroscience information, but that otherwise do not differ in content or logic. In particular, they planned to examine people's ability to distinguish between bad and good explanations and any influence of neuroscience information on this ability. The authors explain: "If logically irrelevant information affects people's judgement of explanations, this would suggest that people's fascination with neuropsychological explanations may stem from an inability or unwillingness to critically consider the role that neuroscience information plays in these explanations."

In the first experiment the 81 participants were divided in two groups: 40 subjects were assigned to the "Without Neuroscience" condition and 41 to the "With Neuroscience" condition. The first group of subject examined explanations without neuroscience, the second one examined explanations that contained neuroscience information, instead.

For 18 psychological phenomena, researchers created two types of explanations: good and bad. For "With Neuroscience" conditions they added neuroscience information to the good and bad explanations of the "Without neuroscience" conditions. Subjects had to rate studies explanations in a scale form -3 (very unsatisfying) to +3 (very satisfying).

No differences in performance based on gender or level of education were found. In subjects rating, good explanations were considered as significantly more satisfying than bad explanations. 
The addiction of neuroscience information had considerable effect: explanations with neuroscience information were rated as significantly more satisfying than explanations that did not include neuroscience information. More in detail, bad explanations with neuroscience information had a better rating than those without information. As the authors write: "neuroscience information seems to have the specific effect of making bad explanations look significantly more satisfying than they would without neuroscience."

Since researchers induced participants to think that explanations were given by knowledgeable researchers, they may have considered those less critically than researchers would have liked. In order to test the training and know-how effect on judgement, in experiment 2 the testing group was composed by students in an intermediate-level cognitive neuroscience class. In the researcher's hypothesis, the students' experience and studies could give them a solid background for judgment and well consider and evaluate the impact of extraneous neuroscience information.

Twenty students were enrolled in the study. Students judged good explanations to be more satisfying than bad explanations. As in the previous experiments, there was a significant effect of neuroscience: although trained on neuroscience and considered by researchers critical consumers of neuroscience information, like novices, students judged experiments with neuroscience more satisfying than those without neuroscience information. Moreover, ratings of bad explanations increased reliably more with the addition of neuroscience, than did good explanations. Authors wrote: "Unlike the novices, the students judged that both good explanations and bad explanations were significantly more satisfying when they contained neuroscience, but the bad explanations were judged to have improved more dramatically, based on a comparison of the differences in ratings between explanations with or without neuroscience." In other words, specialized training apparently does not influence the judgement on irrelevant neuroscience information. A conclusion that points out the strong impact of neuroscience on judgement, although the information given was totally insignificant and the explanations were bad.

Experiment 3 involved 48 experts in neuroscience (divided in two groups 1 . With neuroscience and 2. Without neuroscience), who presumably would be able to distinguish between bad and good explanation and to give the right value to additional neuroscience information.

The experiment material and procedures were identical to experiment 1 , with the addition of four questions in order to confirm their know-how. A high level of expertise was verified among participants. Results demonstrated that experts rated good explanations as significantly more satisfying than bad ones but, unlike in the other experiments, neuroscience information did not have an effect on judgment: the subjects rated explanations in the same way, regardless of the presence of neuroscience information. Moreover good explanations with neuroscience were rated less satisfying than those without neuroscience, recognizing the insufficiency of the neuroscience information in the "With Neuroscience" condition.

We gave an extensive description of the research since it well demonstrates the importance of neuroscience information over its real value, regardless of its own role in explanation. The mere mention of a neural process can influence the participants' judgement, encouraging them to judge explanations more favourably, even the bad ones. That's not just an image matter, then. Even if the image is not showed, like in this case, the neuroscience reference looks to be enough for an explanation positive rating. The three experiments analysed subjects' ratings of how satisfying they found the explanations in four conditions. If the distinction between bad and good explanations was recorded in all the groups, the addition of neuroscience information, even if superfluous, can easily lead to a misunderstanding: "logically irrelevant neuroscience information can be seductive - it can have much more of an impact on participants' judgement than it ought to." It is a psychological matter, evidently.

The speculations about the possible origins of this phenomenon could bring us to hypothesize that the more technical the language explanation is, the more "scientific" it looks. In this context, neuroscience itself appears as an indication of goodness. Or, we could suppose that the reductionist fascination be the cause, intended as the trends to reduce psychological phenomena to their lower-level neuroscientific counterparts. A lower level analysis looks effective and connected to a large explanatory system, and hence more insightful.

Other theories listed by authors include the possibility that neuroscience illustrates a connection between the mind and the brain that people implicitly believe not to exist, or not to exist in such a strong way ${ }^{15}$. Neuroscience is associated with powerful visual imagery, which may merely attract attention to neuroscience studies but which is also known to interfere with subjects' abilities to explain the workings of physical systems and to render scientific claims more convincing. In any case Weisberg et al. conclude their article saying "we have shown that people seem all too ready to accept explanations that 
allude to neuroscience, even if they are not accurate reflections of the scientific data, and even if they would otherwise be seen as far less satisfying [...] even if expert practitioners can easily distinguish good neuroscience explanations from bad, they must not assume that those outside the discipline will be discriminating."

\section{A practical experience: brain research and its consequences on ordinary lives}

A Brazilian study published in 2002 pointed out some interesting evidences. In particular it showed that the public is uninformed about major research methodologies such as electroencephalography or fMRI. ${ }^{16}$ At the same time many researchers and scholars have underlined as simplistic understanding of neuroscience and neuroimaging concerns may raise the risk for misuse and the possibility of the abuse of consumers who are tempted by the technology high-tech profile..$^{17,18,19,20}$

In 2006, Racine, Bar-Ilan and Illes wrote that neuroscience issues have not yet been brought to the public eye as frequently as issues of genomics. That's our perception, too. Although the debate on neuroscience use, or misuse, the ethical aspects, as well as the social ones, are taking up pages on scientific journal, newspapers, and magazines, the most controversial aspects of this technology seem to be still quite far from general debate involving all society branches as, in the past, happened for genomics applications.

Among the projects dedicated to European citizens' involvement on neuroscience instead, stand out the "meeting of minds" initiative, launched by 12 technology assessment bodies, science museums, academic institutions and public foundations form nine European countries. 126 citizens from the different countries were involved, while no professionals in the field of brain science were invited to sit on this European citizens' panel. "To help citizens begin assessing the complex matters of brain science, they were provided with a set of case studies. The case studies were meant to encourage them to reflect and contemplate- without stumbling over technical and scientific hurdles. These cases vividly illustrate how new developments in brain research might affect ordinary lives."

Many recommendations were developed and brought to the EU attentions from participants. The ethic control was one of the focal points. The final recommendations were $37^{21}$ and, looking at those, the first evidence emerging is related to the wide gap existing between brain research and its applications and public information around those themes. So that, groups of participants involved, underlined the need of higher level of information, fundamental to guarantee the freedom in choices and to develop a correct public regulation. The improvement of the public knowledge and awareness about those themes is one of most important aims of future initiatives, since even the public authorities don't have information or databases on the mental health issues. Looking at the research data, neurodegenerative diseases are increasing in incidence, and the estimations and calculations say that in the next future wider society brackets will be affected. These worrying expectations underline the necessity of a good prevention plan that should minimize the social and economic impact of future patients in a difficult balance of social interests and the individual needs of a sick person.

It is a general opinion that in the next future individual needs and necessities will collide with the social organization assistance and their costs. This conflict will improve the problems related to self decision and self determination and the common good. The European dimension is seen as a desirable aim since an extra-national legislation could do a better coordination and management around brain diseases.

As emphasized in the recommendations of the "Meeting of minds", the necessity of a higher public involvement into the debate on neuroscience researches and its medical applications appears more and more important. Citizens' participation in the debate and in results discussion, in particular, should be improved in order to extend the dialogue beyond the boundaries of academic or private research.

\section{Conclusion}

As our collection process strongly underlined, if a quite rich literature dedicated to analyze the persuasive potential of brain imaging images was found, studies strictly focused on citizens' perception are lacking. In our work, with few outstanding exceptions (i.e. "Meeting on minds" project), we didn't find so many projects or researches that collected the public opinion on this matter in the European context. Probably, as some researchers pointed out, because the debate on neuroscience techniques 
applications and their ethical and social consequences is still confined to narrow boundaries, involving scientists, on one side, and communicators, on the other. There is no doubt that interest on brain research is increasing. The number of publications and lay literature that focus on these themes can be a good confirmation of that. At the same time, if the brain fascination is mounting, a critical look that considers the benefits as well as the risks seems to be absent. As we underline in our report, analyzing the existing literature, the discussion between these two categories is extensive and, in some case, characterized by hard tones. Under the fire, there is, in particular, the use (or the misuse) of brain imaging outside of medical applications field. Media communication on brain imaging frequently focus on nonclinical research (studies on higher order cognition and emotion), leading to a possible misinterpretation of results.

We analyzed some recent cases in which the debate and disagreement between media and scientists, and between scientists too, emerged. We analyzed the main researches performed in the last few years exploring the media coverage. We considered, too, the studies dedicated to investigate the persuasive power of brain images on different publics. At the end of the report we took in consideration the project "Meeting of minds" results as a virtuous example of non professionals' engagement in the debate on neuroscience. On the basis of these poor results, any consideration could be judged premature. In this panorama, the necessity of further studies and projects that strongly involve citizens in the discussion about the complex matters of brain science is coming more and more urgent. That is an essential requirement that must be pointed out to UE authorities in order to avoid that, in the next future, what happened in the past with genetics matter (OGM debate above all) could happen again.

\section{Notes and bibliography}

1 G. Miller, Growing Pains for fMRI, Science 320: 1412-1414.

2 E. Racine, O. Bar-Ilan and J. Illes (2005), fMRI in the public eye, Nat. Rev. Neurosci. 6(2): 159-164.

3 E. Racine, O. Bar-Ilan and J. Illes (2006), Brain Imaging A decade of coverage in Print Media, Science Communication 28(1): 122-143.

4 P. Conrad, Genetic optimism: Framing genes and mental illness in the news, Culture, Medicine, and Psychiatry $25(2): 225.47$.

5 D.P. McCabe and A.D. Casterl, Seeing is believing: The effect of brain images ..., Cognition (2007).

6 B. Carey, Searching for the person in the brain, New York Times, $5^{\text {th }}$ of February 2006.

7 C. Umiltà, Un garbuglio di sinapsi, nòva, Il Sole 24 Ore, 27/9/09, pag. 9.

8 D.S. Weisberg, F.C. Keil, J. Goodstein, E. Rawson and J.R. Gray, The Seductive Allure of Neuroscience Explanations, Journal of Cognitive Neuroscience 20(3): 470-477.

9 J.D. Trout, Scientific explanation and the sense of understanding, Phylosophy of Science 69: 212-233.

${ }^{10}$ E. Kikas, University students conception of different physical phenomena, Journal of Adult development 10: 139-150.

${ }^{11}$ L.J. Rips (2002), Circular reasoning, Cognitive Science 26: 767-795.

${ }^{12}$ L. Rosenblit and F. Keil, The misunderstood limits of folk science: An illusion of explanatory depth, Cognitive Science 92: 1-42.

${ }^{13}$ T. Lombrozo and S. Carey, Functional explanation and the function of explanation, Cognition 99: 167-204.

${ }^{14}$ D. Kelemen (1999), Function, goals, and intentio: Children's teleological reasoning about objects, Trends in Cognitive Sciences 3: 461-468.

${ }^{15}$ P. Bloom (2004), Descartes' baby, New York, Basic Books.

${ }^{16}$ S. Herculano-Houzel (2002), Do you know your brain? A survey on public neuroscience literacy at the closing of the decade of the brain, Neuroscientist 8(2): 98-110.

${ }^{17}$ M.J. Farah and P.R. Wolpe (2004), Monitoring and manipulating brain funciont: New neuroscience technologies and their ethical implications, Hastngs Center Report 34(3): 35-45.

${ }^{18}$ T. Gura (2005), Big plans for little brains, Nature 435(7046): 1156-58.

${ }^{19}$ V.J. Hinton (2002), Ethics of neuroimaging in pediatric development, Brain and Cognition 50(3): 455-68.

20 J. Kulynych (2002), Legal and ethical issues in neuroimaging research: Human subject protection, medical privacy, and the public communication of research results, Brain and Cognition 50(3): 345.57.

${ }^{21}$ European Citizens' Assessment report, Meeting of mind Partner Consortium, June 2006.

How TO CITE: D. Ramani, The brain seduction: the public perception of neuroscience, Jcom $\mathbf{0 8}(04)$ (2009) L01 